\title{
SUSY charged Higgs boson pair production via gluon-gluon collisions
}

\author{
Jiang $\mathrm{Yi}^{b}$, Ma Wen-Gan ${ }^{a, b, c},{ }^{*} \mathrm{Han} \mathrm{Liang}^{b}$, Han $\mathrm{Meng}^{b}$ and Yu Zeng-Hui ${ }^{b}$ \\ ${ }^{a}$ CCAST (World Laboratory), P.O.Box 8730, Beijing 100080, P.R.China. \\ ${ }^{b}$ Department of Modern Physics, University of Science and Technology of China (USTC) \\ Hefei, Anhui 230027, P.R.China ! \\ ${ }^{c}$ Institute of Theoretical Physics, Academia Sinica, P.O.Box 2735, Beijing 100080, P.R.China.
}

\begin{abstract}
The production of charged Higgs boson pairs from gluongluon fusion is studied in the minimal supersymmetric model(MSSM) at proton-proton colliders. We find that the contribution of the scalar quark loops to the subprocess production rate is substantial and consequently the production rate in proton-proton colliders is enhanced in the MSSM if there exist scalar quarks with favorable masses. The results show that this cross section may reach a few femptobarn in the future LHC collider with plausible values of the parameters.
\end{abstract}

PACS number(s): 14.80.Cp, 12.15.Lk, 12.60.Jv

*e-mail address: mawg@lx04.mphy.ustc.edu.cn

${ }^{\dagger}$ Mailing address 


\section{Introduction}

The minimal standard model(MSM) [1][2], a gauge theory based on $S U(3)_{c} \times S U(2)_{L} \times$ $U(1)_{Y}$, is a very successful model which nearly describes either quantitatively or qualitatively all available data pertaining to the strong and electroweak interaction phenomena. Until now the symmetric breaking sector of the electroweak interactions has not yet been directly tested experimentally. The future multi-TeV hadron colliders such as the CERN Large Hadron Collider (LHC) and Next Linear Collider(NLC) are elaborately designed in order to detect a 'light' scalar boson $\left(m_{H} \leq 1 \mathrm{TeV}\right)$ associated with the symmetry-breaking mechanism.

Any enlargement of the sector beyond the single $S U(2)_{L}$ Higgs doublet of MSM, with two or more doublets as required in supersymmetric theory, necessarily involves new physical particles. In this extension theory the physical spectrum includes charged Higgs bosons. Like the general two-Higgs-doublet model(THDM), the minimal supersymmetric standard model(MSSM) also requires two $S U(2)$ doublets to give masses to up and down quarks [3] 迎. Since both models contain the same Higgs sector structure, it is of interest to determine whether THDM or MSSM Higgs sector might appear in future experiments.

With regard to colliders in the future there are several mechanisms which can produce charged Higgs bosons. Among them the charged Higgs boson pair productions via gluongluon collision, quark-antiquark annihilation and $\gamma \gamma$ collision, play a significant role in studying charged Higgs bosons. Similar to the neutral Higgs pair production process in hadron colliders, the charged Higgs pair production process also involves Higgs sector interactions, therefore the measurement of these kinds of couplings could be a good test of our theory. 
Recently Plehn et al provided calculations concerning the pair production of neutral Higgs particles in gluon-gluon collisions in MSSM[5] and charged Higgs boson pair production at NLC in $\gamma \gamma$ modes are also investigated in references [6]. The charged Higgs boson pair production via gluon-gluon fusion in general THDM is also represented in Ref. [7]. In this reference, it is concluded that the production rate of this process in THDM is not sensitive to the neutral Higgs boson masses and the couplings of self interactions of Higgs bosons, but it is strongly related to the couplings of the charged Higgs boson to quarks. Willenbrock discussed the charged Higgs boson pair production via gluon fusion in MSSM in Ref. [9]. He showed that the gluon fusion via quark and scalar-quark loops would dominate the usual electroweak quark pair annihilation process if there exist sufficiently heavy quarks or scalarquarks. In his work, he took some approximations, such as the large quark and squark mass limits, i.e., $m_{q}^{2}\left(m_{\tilde{q}}^{2}\right)>>\hat{s}$, in numerical calculations. After we have finished our paper, we found a work also on this process. One can refer to Ref. [10].

In this paper we concentrate on the capability of the charged Higgs boson pair production via gluon-gluon collisions at $p p$ colliders in frame of MSSM without the approximations used in reference [9]. The paper is organized as follows: The analytical evaluations are given in section 2. In section 3 there are numerical results, discussion and a short summary. Finally the explicit expressions of the relevant form factors induced by the supersymmetric quark loop diagrams are collected in Appendix.

\section{Calculation}


The MSSM theory gives a large number of parameters, since it predicts more than doubling the MSM spectrum of particle states. The number of the parameters can be reduced largely by embedding the low-energy supersymmetric theory into a grand unified(GUT) framework. Besides the parameters which can be achieved in supergravity model [8], we need extra parameters to describe the Higgs sector: one Higgs mass parameter and the two vacuum expectation values of the neutral Higgs fields. We can also use $\tan \beta=\frac{v_{2}}{v_{1}}$, the ratio of the vacuum expectation values of the two-Higgs-doublet fields which break the electroweak symmetry. If we ignore the flavor mixing, the mass matrix of a scalar quark takes the following form [1]:

$$
-\mathcal{L}_{m}=\left(\begin{array}{cc}
\tilde{q}_{L}^{*} & \tilde{q}_{R}^{*}
\end{array}\right)\left(\begin{array}{ll}
m_{\tilde{q}_{L}}^{2} & a_{q} m_{q} \\
a_{q}^{*} m_{q} & m_{\tilde{q}_{R}}^{2}
\end{array}\right)\left(\begin{array}{c}
\tilde{q}_{L} \\
\tilde{q}_{R}
\end{array}\right)
$$

here $\tilde{q}_{L}$ and $\tilde{q}_{R}$ are the current eigenstates and for the up-type scalar quarks,

$$
\begin{gathered}
m_{\tilde{q}_{L}}^{2}=\tilde{M}_{Q}^{2}+m_{Z}^{2} \cos 2 \beta\left(\frac{1}{2}-Q_{q} \sin \theta_{W}^{2}\right)+m_{q}^{2} \\
m_{\tilde{q}_{R}}^{2}=\tilde{M}_{U}^{2}+m_{Z}^{2} \cos 2 \beta Q_{q} \sin \theta_{W}^{2}+m_{q}^{2} \\
a_{q}=\mu \cot \beta+A_{q}^{*} \tilde{M}
\end{gathered}
$$

for the down-type scalar quarks,

$$
\begin{gathered}
m_{\tilde{q}_{L}}^{2}=\tilde{M}_{Q}^{2}-m_{Z}^{2} \cos 2 \beta\left(\frac{1}{2}+Q_{q} \sin \theta_{W}^{2}\right)+m_{q}^{2} \\
m_{\tilde{q}_{R}}^{2}=\tilde{M}_{D}^{2}+m_{Z}^{2} \cos 2 \beta Q_{q} \sin \theta_{W}^{2}+m_{q}^{2}, \\
a_{q}=\mu \tan \beta+A_{q}^{*} \tilde{M}
\end{gathered}
$$


where $Q_{q}$ is the charge of the scalar quark, $\tilde{M}_{Q}^{2}, \tilde{M}_{U}^{2}$ and $\tilde{M}_{D}^{2}$ are the self-supersymmetrybreaking mass terms for the left-handed and right-handed scalar quarks. $A_{q} \times \tilde{M}$ is a trilinear scalar interaction parameter, and $\mu$ is the supersymmetric mass mixing term of the Higgs boson. When there is no $\mathrm{CP}$ violation, we can regard that $a_{q}$ is a real constant. At the Plank mass scale we take

$$
\tilde{M}_{Q}^{2}=\tilde{M}_{U}^{2}=\tilde{M}_{D}^{2}=\tilde{M}^{2}
$$

The mass eigenstates $\tilde{q}_{1}$ and $\tilde{q}_{2}$ are related to the current eigenstates $\tilde{q}_{L}$ and $\tilde{q}_{R}$ by

$$
\begin{gathered}
\tilde{q}_{1}=\tilde{q}_{L} \cos \theta_{q}+\tilde{q}_{R} \sin \theta_{q} \\
\tilde{q}_{2}=-\tilde{q}_{L} \sin \theta_{q}+\tilde{q}_{R} \cos \theta_{q} .
\end{gathered}
$$

and

$$
\tan 2 \theta_{q}=\frac{2 a_{q} m_{q}}{m_{\tilde{q}_{R}}^{2}-m_{\tilde{q}_{L}}^{2}}
$$

The masses of $\tilde{q}_{1}$ and $\tilde{q}_{2}$ are

$$
\left(m_{\tilde{q}_{1}}^{2}, m_{\tilde{q}_{2}}^{2}\right)=\frac{1}{2} m_{\tilde{q}_{L}}^{2}+m_{\tilde{q}_{R}}^{2} \mp\left[\left(m_{\tilde{q}_{L}}^{2}-m_{\tilde{q}_{R}}^{2}\right)^{2}+4 a_{q}^{2} m_{q}^{2}\right]^{\frac{1}{2}} .
$$

In the evaluation we neglected the first SUSY generation mixing angle, whereas in the second SUSY generation we took $\sin \theta_{q}=0.2$, and let the third SUSY generation has the mixing with $\sin \theta_{q} \approx \frac{1}{\sqrt{2}}$ due to the large splitting of the mass eigenstates. The numerical value of the lightest physical stop quark mass is taken as $m_{\tilde{t}_{1}}=180 \mathrm{GeV}$ and the sbottom 
quark mass as $m_{\tilde{b}_{1}}=400 \mathrm{GeV}$. We set the masses of the squarks of $\tilde{u}_{1}, \tilde{d}_{1}, \tilde{c}_{1}$ and $\tilde{s}_{1}$ degenerated with mass value $800 \mathrm{GeV}$. As a quantitative example, we took $\mu=0$ in our calculation.

The generic Feynman diagrams contributing to the subprocess $g g \rightarrow H^{+} H^{-}$which are involved in frame of MSSM are depicted in figure 1. The diagrams created by exchanging the two external gluon lines are not shown in Fig.1. Fig.1 $(1 \sim 10)$ are the same as those in the THDM model[7], we call them as "THDM part". Fig.1(11 32) are the diagrams with squark-loop. There is no tree level contribution for this subprocess, therefore the proper vertex counterterm cancels with the counterterms of the external legs diagrams. That is to say that the evolution can be simply carry out by summing all unrenormalized reducible and irreducible diagrams. The result is finite and gauge invariant. The relevant Feynman rules can be found in references[1] [12. Since the main contributions are due to the loops of heavy quarks and the scalar quarks, we neglect the light quark loop diagrams. We can see from Fig.1 that there are mainly three kinds of production mechanisms. One is by exchanging a photon or a $Z^{0}$ boson to produce a pair of charged Higgs bosons, we refer to this as $\gamma-Z^{0}$ exchange s-channel (see Fig.1(1,2), Fig.1(11,12), Fig.1(15,16) and Fig.1(19,20)). For the $\gamma-Z^{0}$ exchange s-channel diagrams, the contributions are shown to be zero. This is the consequence of Furry theorem. Since the Furry theorem forbids the production of the spin-one components of the $Z^{0}$ and $\gamma$, and the spin-zero component of the $Z^{0}$ vector boson does not couple to a pair of scalar particles with the same mass. The second mechanism is through producing the virtual neutral Higgs $h^{0}$ and $H^{0}$ bosons which are coupled to 
gluons by quartic coupling including squarks or by a triangle of heavy quarks or squarks, then the charged Higgs boson pair is appeared by the subsequent decay of the virtual Higgs bosons (see Fig.1(3,4,13,14,17,18)). It is called neutral Higgs boson exchange s-channel. An alternative is that the charged Higgs bosons are produced by means of the box and quartic interactions.

In this paper we follow the notations in reference [0] that $p_{1}, p_{2}, p_{3}$ and $p_{4}$ represent the four-momenta of the final charged Higgs bosons and the initial gluons, respectively. We write the corresponding matrix element for each of the diagrams in Fig.1 in the form as

$$
\begin{aligned}
& M_{l}= \frac{-1}{16 \pi^{2}} g_{s}^{2} \epsilon_{\mu}\left(p_{3}\right) \epsilon_{\nu}\left(p_{4}\right) A_{2} \cdot \\
&\left(f_{1, l} g^{\mu \nu}+f_{2, l} p_{1}^{\mu} p_{1}^{\nu}+f_{3, l} p_{1}^{\mu} p_{2}^{\nu}+f_{4, l} p_{2}^{\mu} p_{1}^{\nu}+f_{5, l} p_{2}^{\mu} p_{2}^{\nu}+\right. \\
& f_{6, l} \epsilon^{\mu \nu \alpha \beta} p_{1 \alpha} p_{2 \beta}+f_{7, l} \epsilon^{\mu \nu \alpha \beta} p_{1 \alpha} p_{3 \beta}+f_{8, l} \epsilon^{\mu \nu \alpha \beta} p_{2 \alpha} p_{3 \beta}+ \\
& f_{9, l} \epsilon^{\nu \alpha \beta \gamma} p_{1 \alpha} p_{2 \beta} p_{3 \gamma} p_{1}^{\mu}+f_{10, l} \epsilon^{\mu \alpha \beta \gamma} p_{1 \alpha} p_{2 \beta} p_{3 \gamma} p_{1}^{\nu}+ \\
&\left.f_{11, l} \epsilon^{\nu \alpha \beta \gamma} p_{1, \alpha} p_{2, \beta} p_{3, \gamma} p_{2}^{\mu}+f_{12, l} \epsilon^{\mu \alpha \beta \gamma} p_{1, \alpha} p_{2, \beta} p_{3, \gamma} p_{2}^{\nu}\right) .
\end{aligned}
$$

We denote $A_{k}(\mathrm{k}=1,2)$ as the color factors of the diagrams with and without gluon-gluonsquark-squark quartic vertex respectively and it can be expressed as

$$
\begin{gathered}
A_{1}=\operatorname{Tr}\left[\frac{1}{3} \delta_{a b} I+d_{a b c} T^{c}\right]=\delta_{a b} \\
A_{2}=\operatorname{Tr}\left[T^{a} T^{b}\right]=\frac{1}{2} \delta_{a b}
\end{gathered}
$$

In above equations $I$ is a $3 \times 3$ unit matrix. $g_{s}$ is the strong coupling constant and $T^{a}(a=1 \sim 8)$ are the $S U(3)_{c}$ generators introduced by Gell-Mann and $d_{a b c}$ are the structure 
constants 13]. Here we should declare that in Eq.(8) $A_{2}=\frac{1}{2} \delta_{a b}$ is the common factor of the all color factors in amplitudes and the remained parts of color factors are collected in form factors.

The total matrix element of subprocess $g g \rightarrow H^{+} H^{-}$in MSSM can be expressed as

$$
\begin{aligned}
M & =\sum_{l=1}^{10} M_{l}+\sum_{n=11}^{32} M_{n} \\
& =M_{T H D M}+M_{S U S Y},
\end{aligned}
$$

where $M_{T H D M}$ represents the summation of the amplitudes of the THDM part and $M_{S U S Y}$ is the total amplitudes of squark-loop diagrams.

Then we can split each form factor appeared in total matrix element up into two parts.

$$
f_{i}=f_{i}^{T H D M}+f_{i}^{S U S Y},(i=1 \sim 12)
$$

where $f_{i}^{S U S Y}(i=1 \sim 12)=\sum_{n=11}^{32} f_{i, n}$ and $f_{i}^{T H D M}(i=1 \sim 12)=\sum_{l=1}^{10} f_{i, l}$. The explicit expressions of the form factors $f_{i}^{S U S Y}(i=1 \sim 12)$, which come from the squark-loop diagrams, are listed in Appendix. For the form factors of the THDM part(i.e. $\left.f_{i}^{T H D M}(i=1 \sim 12)\right)$ one can find them in reference[7]. The total cross section for subprocess $g g \rightarrow H^{+} H^{-}$can finally be written in the form

$$
\hat{\sigma}(\hat{s})=\frac{1}{16 \pi \hat{s}^{2}} \int_{\hat{t}^{-}}^{\hat{t}^{+}} d \hat{t}|\bar{M}|^{2}
$$

where $|\bar{M}|^{2}$ is the initial spin and color averaged matrix element squared and $\hat{t}^{ \pm}=\left(m_{H^{ \pm}}^{2}-\right.$ $\left.\frac{1}{2} \hat{s}\right) \pm \frac{1}{2} \hat{s} \beta_{H^{ \pm}}$. The total cross section for the charged Higgs pair production through gluongluon fusion in proton-proton collisions can be obtained by integrating the $\hat{\sigma}$ over the gluon luminosity.

$$
\sigma\left(p p \rightarrow g g \rightarrow H^{+} H^{-}+X\right)=\int_{4 m_{H^{ \pm}}^{2} / s}^{1} d \tau \frac{d L_{g g}}{d \tau} \hat{\sigma}\left(g g \rightarrow H^{+} H^{-} \quad \text { at } \quad \hat{s}=\tau s\right)
$$


where $\sqrt{s}$ and $\sqrt{\hat{s}}$ denote the proton-proton and gluon-gluon c.m. energies respectively and $\frac{d L_{g g}}{d \tau}$ is the gluon luminosity, which is defined as

$$
\frac{d \mathcal{L}_{g g}}{d \tau}=\int_{\tau}^{1} \frac{d x_{1}}{x_{1}}\left[F_{g}\left(x_{1}, \mu\right) F_{g}\left(\frac{\tau}{x_{1}}, \mu\right)\right]
$$

Here we used $\tau=x_{1} x_{2}$, and one can find the definitions of $x_{1}$ and $x_{2}$ in Ref.[7]. In our

calculation we adopt the MRS set G parton distribution function $F_{g}(x)$ [14], and ignore the supersymmetric QCD corrections to the parton distribution functions for simplicity. The numerical calculation is carried out for the LHC at the energy around $10 \sim 14 T e V$.

\section{Numerical results and discussion}

For the numerical evaluation we take the input parameters 15 as $m_{b}=4.5 \mathrm{GeV}, m_{Z}=$ $91.1887 \mathrm{GeV}, m_{W}=80.2226 \mathrm{GeV}, m_{t}=175 \mathrm{GeV}, G_{F}=1.166392 \times 10^{-5}(\mathrm{GeV})^{-2}$ and $\alpha=$ $\frac{1}{137.036}$. We adopt a simple one-loop formula for the running strong coupling constant $\alpha_{s}$ as

$$
\alpha_{s}(\mu)=\frac{\alpha_{s}\left(m_{Z}\right)}{1+\frac{33-2 n_{f}}{6 \pi} \alpha_{s}\left(m_{Z}\right) \ln \left(\frac{\mu}{m_{Z}}\right)} .
$$

where $\alpha_{s}\left(m_{Z}\right)=0.117$ and $n_{f}$ is the number of active flavors at energy scale $\mu$. The two representative quantities of the two vacuum expectation values $\tan \beta=1.5$ and $\tan \beta=30$ are chosen.

We take the mass of charged Higgs bosons $m_{H^{ \pm}}$, and the ratio of vacuum expectation values as free parameters in MSSM. The other Higgs boson masses and the mixing angle $\alpha$ are fixed by quoting the MSSM relations in terms of $\tan \beta$ and $m_{H^{ \pm}}$. The relevant formulas are given as 


$$
\begin{gathered}
\tan 2 \alpha=\tan 2 \beta \frac{m_{A}^{2}+m_{Z}^{2}}{m_{A}^{2}-m_{Z}^{2}}, \text { where } m_{A}^{2}=m_{H^{ \pm}}^{2}-m_{W}^{2} . \\
m_{H^{0}, h^{0}}^{2}=\frac{1}{2}\left[m_{A}^{2}+m_{Z}^{2} \pm \sqrt{\left(m_{A}^{2}+m_{Z}^{2}\right)^{2}-4 m_{Z}^{2} m_{A}^{2} \cos ^{2} 2 \beta}\right] .
\end{gathered}
$$

The cross sections of the subprocess $g g \rightarrow H^{+} H^{-}$with $m_{H^{ \pm}}=150 \mathrm{GeV}$ are depicted in Fig.2(1), on the conditions of the mass values of squarks as mentioned above. In this figure, the small peak around $\sqrt{\hat{s}} \sim 350 \mathrm{GeV}$ of each curve originates from the resonance effect, where $\sqrt{\hat{s}} \sim 2 m_{t}$ and $\sqrt{\hat{s}} \sim 2 m_{\tilde{t}_{1}}$ are satisfied. Around $\sqrt{\hat{s}} \sim 800 \mathrm{GeV}$, there is another higher peak for each curve, which comes from the enhancement of the resonance effects where the c.m. energy of gluon-gluon system is located at $\sqrt{\hat{s}} \sim 2 m_{\tilde{t}_{2}} \sim 800 \mathrm{GeV}$ and $\sqrt{\hat{s}} \sim 2 m_{\tilde{b}_{1}} \sim 800 \mathrm{GeV}$. Fig.2(2) shows the cross section of this subprocess as the functions of c.m. energy of incoming gluons with the same neutral Higgs boson, quark and squark masses as in Fig.2(1), but $m_{H^{ \pm}}=300 \mathrm{GeV}$. In Fig.2(2) again the resonance effects of the squarks are obvious at the position near $\sqrt{\hat{s}} \sim 800 \mathrm{GeV}$.

The dependence of the subprocess cross section to the ratio of vacuum expectation values, is plotted in Fig.3 with $m_{H^{+}}=150 \mathrm{GeV}$. The two curves correspond to $\sqrt{\hat{s}}=400 \mathrm{GeV}$ and $\sqrt{\hat{s}}=800 \mathrm{GeV}$, respectively. They all have the minimal cross sections when $\tan \beta$ is around 6.5. The figure shows that the cross section of the subprocess increases when the $\sqrt{\hat{s}}$ goes up from $400 \mathrm{GeV}$ to $800 \mathrm{GeV}$. The dependence of the subprocess cross section to the mass of charged Higgs bosons is shown in Fig.4 with $\sqrt{\hat{s}}=1 T e V$. The cross section increases in the region where the charged Higgs boson mass has the value approximately larger than $\sim 300 \mathrm{GeV}$. That is because when the value of the the charged Higgs boson mass approaches 
$\frac{1}{2} \sqrt{\hat{s}}=500 \mathrm{GeV}$, the production rate will be enhanced by the threshold effect, but when the mass of charged Higgs boson is very close to $500 \mathrm{GeV}$, the cross section drops down quickly due to the phase space suppression. The cross sections of process $p p \rightarrow g g \rightarrow H^{+} H^{-}+X$ in the LHC energy regions in both MSSM and THDM are depicted in Fig.5(1 2). The charged Higgs boson pair production rates in MSSM are read to be about 1.9 to 5.2 femptobarn at the LHC energy regions when $m_{H^{+}}=150 \mathrm{GeV}$. With the heavier charged Higgs mass, the production rate will be smaller. The production rates in the MSSM are higher than those in the THDM with the same parameters. In our calculation, we also find that among the squark-loop diagrams, the third generation squarks make the decisive contribution, while the contribution from the other squarks is rather small comparatively due to the decoupling of the heavy squarks.

In conclusion, we investigated the pair production process of charged Higgs bosons via gluon-gluon fusion in pp collider at LHC. The numerical analysis of its cross section is carried out in the MSSM model. With the possible parameters, the cross sections at future LHC collider may reach 5.2 femptobarn. The calculation shows that the contribution from the squark-loop diagrams is obvious and consequently enhances the cross section of $p p \rightarrow g g \rightarrow$ $H^{+} H^{-}+X$, if there exist scalar quarks with favorable masses.

We express our thanks to Dr. M. Spira for useful discussions. This work was supported by the National Natural Science Foundation of China. Part of this work was done when two of the authors, Ma Wen-Gan and Yu Zeng-Hui, visited the University Vienna under an exchange agreement(project number: IV.B.12). 


\section{Appendix}

The form factors involved in equation (8) are presented explicitly as:

$$
\begin{aligned}
& f_{1}=-2 g_{s}^{2} \sum_{i=1,2}\left\{\sum_{\tilde{q}_{i}=\tilde{u}_{i}, \tilde{d}_{i}, \tilde{c}_{i}}^{\tilde{s}_{i}, \tilde{t}_{i}, \tilde{b}_{i}}\left(g_{h^{0} H^{ \pm} H^{\mp}} g_{h^{0} \tilde{q}_{i} \tilde{q}_{i}} A_{h^{0}}+g_{H^{0} H^{ \pm} H^{\mp}} g_{H^{0} \tilde{q}_{i} \tilde{q}_{i}} A_{H^{0}}+g_{H^{+} H^{-} \tilde{q}_{i} \tilde{q}_{i}}\right)\right. \\
& \left\{4 C_{24}\left[-p_{3}, p_{1}+p_{2}, m_{\tilde{q}_{i}}, m_{\tilde{q}_{i}}, m_{\tilde{q}_{i}}\right]-B_{0}\left[-p_{1}-p_{2}, m_{\tilde{q}_{i}}, m_{\tilde{q}_{i}}\right]\right\} \\
& +2 \sum_{j=1,2} \sum_{\left(\tilde{q}_{u, i}, \tilde{q}_{d, j}\right)=\left(\tilde{u}_{i}, \tilde{d}_{j}\right)}^{\left(\tilde{c}_{i}, \tilde{s}_{j}\right),\left(\tilde{t}_{i}, \tilde{b}_{j}\right)} g_{H^{ \pm} \tilde{q}_{u, i} \tilde{q}_{d, j}}^{2}\left\{-\frac{1}{2} C_{0}\left[p_{1}+p_{2},-p_{2}, m_{\tilde{q}_{u, i}}, m_{\tilde{q}_{u, i}}, m_{\tilde{q}_{d, i}}\right]\right. \\
& -\frac{1}{2} C_{0}\left[p_{1}+p_{2},-p_{2}, m_{\tilde{q}_{d, i}}, m_{\tilde{q}_{d, i}}, m_{\tilde{q}_{u, i}}\right] \\
& +i D_{27}\left[-p_{3}, p_{1}+p_{2},-p_{2}, m_{\tilde{q}_{u, i}}, m_{\tilde{q}_{u, i}}, m_{\tilde{q}_{u, i}}, m_{\tilde{q}_{d, j}}\right] \\
& +i D_{27}\left[-p_{3}, p_{1}+p_{2},-p_{2}, m_{\tilde{q}_{d, j}}, m_{\tilde{q}_{d, j}}, m_{\tilde{q}_{d, j}}, m_{\tilde{q}_{u, i}}\right] \\
& +i D_{27}\left[p_{3},-p_{1}-p_{2}, p_{1}, m_{\tilde{q}_{u, i}}, m_{\tilde{q}_{u, i}}, m_{\tilde{q}_{u, i}}, m_{\tilde{q}_{d, j}}\right] \\
& +i D_{27}\left[p_{3},-p_{1}-p_{2}, p_{1}, m_{\tilde{q}_{d, j}}, m_{\tilde{q}_{d, j}}, m_{\tilde{q}_{d, j}}, m_{\tilde{q}_{u, i}}\right] \\
& +\quad D_{27}\left[p_{1}, p_{2}-p_{3},-p_{2}, m_{\tilde{q}_{d, j}}, m_{\tilde{q}_{u, i}}, m_{\tilde{q}_{d, j}}, m_{\tilde{q}_{u, i}}\right] \\
& \left.\left.+D_{27}\left[p_{1}, p_{2}-p_{3},-p_{2}, m_{\tilde{q}_{u, i}}, m_{\tilde{q}_{d, j}}, m_{\tilde{q}_{u, i}}, m_{\tilde{q}_{d, j}}\right]\right\}\right\} \\
& f_{2}=-2 g_{s}^{2} \sum_{i=1,2}\left\{\sum_{\tilde{q}_{i}=\tilde{u}_{i}, \tilde{d}_{i}, \tilde{c}_{i}}^{\tilde{s}_{i}, \tilde{t}_{i}, \tilde{b}_{i}}\left(g_{h^{0} H^{ \pm} H^{\mp}} g_{h^{0} \tilde{q}_{i} \tilde{q}_{i}} A_{h^{0}}+g_{H^{0} H^{ \pm} H^{\mp}} g_{H^{0} \tilde{q}_{i} \tilde{q}_{i}} A_{H^{0}}+g_{H^{+} H^{-} \tilde{q}_{i} \tilde{q}_{i}}\right)\right. \\
& \left(C_{23}-C_{22}\right)\left[-p_{3}, p_{1}+p_{2}, m_{\tilde{q}_{i}}, m_{\tilde{q}_{i}}, m_{\tilde{q}_{i}}\right] \\
& \left(\tilde{c}_{i}, \tilde{s}_{j}\right),\left(\tilde{t}_{i}, \tilde{b}_{j}\right) \\
& +\sum_{j=1,2} \sum_{\left(\tilde{q}_{u, i}, \tilde{q}_{d, j}\right)=\left(\tilde{u}_{i}, \tilde{d}_{j}\right)} g_{H^{ \pm} \tilde{q}_{u, i} \tilde{q}_{d, j}}^{2}\left\{2 i \left(D_{24}\right.\right. \\
& \left.-D_{22}\right)\left[-p_{3}, p_{1}+p_{2},-p_{2}, m_{\tilde{q}_{u, i}}, m_{\tilde{q}_{u, i}}, m_{\tilde{q}_{u, i}}, m_{\tilde{q}_{d, j}}\right]
\end{aligned}
$$




$$
\begin{aligned}
& +2 i\left(D_{24}-D_{22}\right)\left[-p_{3}, p_{1}+p_{2},-p_{2}, m_{\tilde{q}_{d, j}}, m_{\tilde{q}_{d, j}}, m_{\tilde{q}_{d, j}}, m_{\tilde{q}_{u, i}}\right] \\
& +2 i\left(D_{24}+2 D_{26}-D_{22}-D_{23}-D_{25}\right)\left[p_{3},-p_{1}-p_{2}, p_{1}, m_{\tilde{q}_{u, i}}, m_{\tilde{q}_{u, i}}, m_{\tilde{q}_{u, i}}, m_{\tilde{q}_{d, j}}\right] \\
& +2 i\left(D_{24}+2 D_{26}-D_{22}-D_{23}-D_{25}\right)\left[p_{3},-p_{1}-p_{2}, p_{1}, m_{\tilde{q}_{d, j}}, m_{\tilde{q}_{d, j}}, m_{\tilde{q}_{d, j}}, m_{\tilde{q}_{u, i}}\right] \\
& -\left(D_{0}+3 D_{11}+2 D_{21}\right)\left[p_{1}, p_{2}-p_{3},-p_{2}, m_{\tilde{q}_{d, j}}, m_{\tilde{q}_{u, i}}, m_{\tilde{q}_{d, j}}, m_{\tilde{q}_{u, i}}\right] \\
& \left.\left.-\left(D_{0}+3 D_{11}+2 D_{21}\right)\left[p_{1}, p_{2}-p_{3},-p_{2}, m_{\tilde{q}_{u, i}}, m_{\tilde{q}_{d, j}}, m_{\tilde{q}_{u, i}}, m_{\tilde{q}_{d, j}}\right]\right\}\right\}
\end{aligned}
$$

$$
\begin{aligned}
f_{3}= & -2 g_{s}^{2} \sum_{i=1,2}\left\{\sum_{\tilde{q}_{i}=\tilde{u}_{i}, \tilde{d}_{i}, \tilde{c}_{i}}^{\tilde{s}_{i}, \tilde{t}_{i}, \tilde{b}_{i}}\left(g_{h^{0} H^{ \pm} H^{\mp}} g_{h^{0} \tilde{q}_{i} \tilde{q}_{i}} A_{h^{0}}+g_{H^{0} H^{ \pm} H^{\mp}} g_{H^{0} \tilde{q}_{i} \tilde{q}_{i}} A_{H^{0}}+g_{H^{+} H^{-} \tilde{q}_{\tilde{q}} \tilde{q}_{i}}\right)\right. \\
& \left(C_{23}-C_{22}\right)\left[-p_{3}, p_{1}+p_{2}, m_{\tilde{q}_{i}}, m_{\tilde{q}_{i}}, m_{\tilde{q}_{i}}\right] \\
+ & \sum_{j=1,2} \sum_{\left(\tilde{q}_{u, i}, \tilde{q}_{d, j}\right)=\left(\tilde{u}_{i}, \tilde{s}_{j}\right),\left(\tilde{t}_{i}, \tilde{b}_{j}\right)} g_{H^{ \pm} \tilde{q}_{u, i} \tilde{q}_{d, j}}^{2}\left\{2 i \left(D_{24}+D_{26}\right.\right. \\
& \left.-D_{22}\right)\left[-p_{3}, p_{1}+p_{2},-p_{2}, m_{\tilde{q}_{u, i}}, m_{\tilde{q}_{u, i}}, m_{\tilde{q}_{u, i}}, m_{\tilde{q}_{d, j}}\right] \\
+ & 2 i\left(D_{24}+D_{26}-D_{22}\right)\left[-p_{3}, p_{1}+p_{2},-p_{2}, m_{\tilde{q}_{d, j}}, m_{\tilde{q}_{d, j}}, m_{\tilde{q}_{d, j}}, m_{\tilde{q}_{u, i}}\right] \\
+ & 2 i\left(D_{24}+D_{26}-D_{22}-D_{25}\right)\left[p_{3},-p_{1}-p_{2}, p_{1}, m_{\tilde{q}_{u, i}}, m_{\tilde{q}_{u, i}}, m_{\tilde{q}_{u, i}}, m_{\tilde{q}_{d, j}}\right] \\
+ & 2 i\left(D_{24}+D_{26}-D_{22}-D_{25}\right)\left[p_{3},-p_{1}-p_{2}, p_{1}, m_{\tilde{q}_{d, j}}, m_{\tilde{q}_{d, j}}, m_{\tilde{q}_{d, j}}, m_{\tilde{q}_{u, i}}\right] \\
- & \left(D_{0}+D_{11}+2 D_{12}-2 D_{13}+2 D_{24}-2 D_{25}\right)\left[p_{1}, p_{2}-p_{3},-p_{2}, m_{\tilde{q}_{d, j}}, m_{\tilde{q}_{u, i}}, m_{\tilde{q}_{d, j}}, m_{\tilde{q}_{u, i}}\right] \\
- & \left.\left.\left(D_{0}+D_{11}+2 D_{12}-2 D_{13}+2 D_{24}-2 D_{25}\right)\left[p_{1}, p_{2}-p_{3},-p_{2}, m_{\tilde{q}_{u, i}}, m_{\tilde{q}_{d, j}}, m_{\tilde{q}_{u, i}}, m_{\tilde{q}_{d, j}}\right]\right\}\right\}
\end{aligned}
$$

$$
\begin{aligned}
f_{4}= & -2 g_{s}^{2} \sum_{i=1,2}\left\{\sum_{\tilde{q}_{i}=\tilde{u}_{i}, \tilde{d}_{i}, \tilde{c}_{i}}^{\tilde{s}_{i}, \tilde{t}_{i}, \tilde{b}_{i}}\left(g_{h^{0} H^{ \pm} H^{\mp}} g_{h^{0} \tilde{q}_{i} \tilde{q}_{i}} A_{h^{0}}+g_{H^{0} H^{ \pm} H^{\mp}} g_{H^{0}} \tilde{q}_{i} \tilde{q}_{i} A_{H^{0}}+g_{H^{+} H^{-} \tilde{q}_{i} \tilde{q}_{i}}\right)\right. \\
& \left(C_{23}-C_{22}\right)\left[-p_{3}, p_{1}+p_{2}, m_{\tilde{q}_{i}}, m_{\tilde{q}_{i}}, m_{\tilde{q}_{i}}\right]
\end{aligned}
$$




$$
\begin{aligned}
& +\sum_{j=1,2} \sum_{\left(\tilde{q}_{u, i}, \tilde{q}_{d, j}\right)=\left(\tilde{u}_{i}, \tilde{d}_{j}\right)}^{\left(\tilde{c}_{i}, \tilde{s}_{j}\right),\left(\tilde{t}_{i}, \tilde{b}_{j}\right)} g_{H^{ \pm} \tilde{q}_{u, i} \tilde{q}_{d, j}}^{2}\left\{2 i \left(D_{24}+D_{26}\right.\right. \\
& \left.\quad-D_{22}\right)\left[-p_{3}, p_{1}+p_{2},-p_{2}, m_{\tilde{q}_{u, i}}, m_{\tilde{q}_{u, i}}, m_{\tilde{q}_{u, i}}, m_{\tilde{q}_{d, j}}\right] \\
& +\quad 2 i\left(D_{24}+D_{26}-D_{22}\right)\left[-p_{3}, p_{1}+p_{2},-p_{2}, m_{\tilde{q}_{d, j}}, m_{\tilde{q}_{d, j}}, m_{\tilde{q}_{d, j}}, m_{\tilde{q}_{u, i}}\right] \\
& +\quad 2 i\left(D_{24}+D_{26}-D_{22}-D_{25}\right)\left[p_{3},-p_{1}-p_{2}, p_{1}, m_{\tilde{q}_{u, i}}, m_{\tilde{q}_{u, i}}, m_{\tilde{q}_{u, i}}, m_{\tilde{q}_{d, j}}\right] \\
& +\quad 2 i\left(D_{24}+D_{26}-D_{22}-D_{25}\right)\left[p_{3},-p_{1}-p_{2}, p_{1}, m_{\tilde{q}_{d, j}}, m_{\tilde{q}_{d, j}}, m_{\tilde{q}_{d, j}}, m_{\tilde{q}_{u, i}}\right] \\
& -\quad\left(D_{12}-D_{13}+2 D_{24}-2 D_{25}\right)\left[p_{1}, p_{2}-p_{3},-p_{2}, m_{\left.\tilde{q}_{d, j}, m_{\tilde{q}_{u, i}}, m_{\tilde{q}_{d, j}}, m_{\tilde{q}_{u, i}}\right]}+\left(D_{12}-D_{13}+2 D_{24}-2 D_{25}\right)\left[p_{1}, p_{2}-p_{3},-p_{2}, m_{\tilde{q}_{u, i}}, m_{\tilde{q}_{d, j}}, m_{\tilde{q}_{u, i}}, m_{\tilde{q}_{d, j}}\right]\right\}
\end{aligned}
$$

$$
\begin{aligned}
f_{5}= & -2 g_{s}^{2} \sum_{i=1,2}\left\{\sum_{\tilde{q}_{i}=\tilde{u}_{i}, \tilde{d}_{i}, \tilde{c}_{i}}^{\tilde{s}_{i}, \tilde{t}_{i}, \tilde{b}_{i}}\left(g_{h^{0} H^{ \pm} H^{\mp}} g_{h^{0} \tilde{q}_{i} \tilde{q}_{i}} A_{h^{0}}+g_{H^{0} H^{ \pm} H^{\mp}} g_{H^{0} \tilde{q}_{i} \tilde{q}_{i}} A_{H^{0}}+g_{H^{+} H^{-} \tilde{q}_{i} \tilde{q}_{i}}\right)\right. \\
& \left(C_{23}-C_{22}\right)\left[-p_{3}, p_{1}+p_{2}, m_{\tilde{q}_{i}}, m_{\tilde{q}_{i}}, m_{\tilde{q}_{i}}\right] \\
+ & \sum_{j=1,2} \sum_{\left(\tilde{c}_{i}, \tilde{s}_{j}\right),\left(\tilde{t}_{i}, \tilde{b}_{j}\right)} g_{\left.\tilde{q}_{d, j}\right)=\left(\tilde{u}_{i}, \tilde{d}_{j}\right)}^{2} g_{\tilde{q}_{u, i} \tilde{q}_{d, j}}\left\{2 i \left(D_{24}\right.\right. \\
& \left.-D_{22}\right)\left[-p_{3}, p_{1}+p_{2},-p_{2}, m_{\tilde{q}_{u, i}}, m_{\tilde{q}_{u, i}}, m_{\tilde{q}_{u, i}}, m_{\tilde{q}_{d, j}}\right] \\
+ & 2 i\left(D_{24}-D_{22}\right)\left[-p_{3}, p_{1}+p_{2},-p_{2}, m_{\tilde{q}_{d, j}}, m_{\tilde{q}_{d, j}}, m_{\tilde{q}_{d, j}}, m_{\tilde{q}_{u, i}}\right] \\
+ & 2 i\left(D_{24}+2 D_{26}-D_{22}-D_{23}-D_{25}\right)\left[p_{3},-p_{1}-p_{2}, p_{1}, m_{\tilde{q}_{u, i}}, m_{\tilde{q}_{u, i}}, m_{\tilde{q}_{u, i}}, m_{\tilde{q}_{d, j}}\right] \\
+ & 2 i\left(D_{24}+2 D_{26}-D_{22}-D_{23}-D_{25}\right)\left[p_{3},-p_{1}-p_{2}, p_{1}, m_{\tilde{q}_{d, j}}, m_{\tilde{q}_{d, j}}, m_{\tilde{q}_{d, j}}, m_{\tilde{q}_{u, i}}\right] \\
- & \left(D_{12}-D_{13}+2 D_{22}+2 D_{23}-4 D_{26}\right)\left[p_{1}, p_{2}-p_{3},-p_{2}, m_{\tilde{q}_{d, j}}, m_{\tilde{q}_{u, i}}, m_{\tilde{q}_{d, j}}, m_{\tilde{q}_{u, i}}\right] \\
& \left.-\left(D_{12}-D_{13}+2 D_{22}+2 D_{23}-4 D_{26}\right)\left[p_{1}, p_{2}-p_{3},-p_{2}, m_{\tilde{q}_{u, i}}, m_{\tilde{q}_{d, j}}, m_{\tilde{q}_{u, i}}, m_{\tilde{q}_{d, j}}\right]\right\}
\end{aligned}
$$




$$
\begin{gathered}
f_{6}=f_{7}=\cdots=f_{12}=0 \\
A_{H^{0}}=\frac{i}{s-m_{H^{0}}^{2}+i \Gamma_{H^{0}} m_{H^{0}}}, \\
A_{h^{0}}=\frac{i}{s-m_{h^{0}}^{2}+i \Gamma_{h^{0}} m_{h^{0}}} .
\end{gathered}
$$

The coupling constants are obtained by converting Feynman rules for $X \tilde{q} \tilde{q}$ (where $\mathrm{X}$ is a oneor two-particle state) from the $\tilde{q}_{L}-\tilde{q}_{R}$ basis, their Feynman rules can be found in Ref.[18], to the $\tilde{q}_{1}-\tilde{q}_{2}$ basis. For the converting formulae one can refer to reference 4 .

In above expressions we adopted the definitions of one-loop integral functions in reference [16] and defined $d=4-2 \epsilon$. The arguments of two-point, three-point and four-point integral functions are written at the end of formulae in brackets. The numerical calculation of the vector and tensor loop integral functions can be traced back to four scalar loop integrals $A_{0}$, $B_{0}, C_{0}, D_{0}$ as shown in the reference 17 . 


\section{References}

[1] S.L. Glashow, Nucl. Phys. 22(1961)579; S. Weinberg, Phys. Rev. Lett. 1(1967)1264; A. Salam, Proc. 8th Nobel Symposium Stockholm 1968, ed. N. Svartholm(Almquist and Wiksells, Stockholm 1968) p.367; H.D. Politzer, Phys. Rep. 14(1974)129.

[2] P.W. Higgs, Phys. Lett 12(1964)132, Phys. Rev. Lett. 13 (1964)508; Phys.Rev. 145(1966)1156; F.Englert and R.Brout, Phys. Rev. Lett. 13(1964)321; G.S. Guralnik, C.R.Hagen and T.W.B. Kibble, Phys. Rev. Lett. 13(1964)585; T.W.B. Kibble, Phys. Rev. 155(1967)1554.

[3] H. E. Haber, G. L. Kane, Phys. Rep. 117(1985) 75.

[4] J.F. Gunion and H.E. Haber, Nucl. Phys. B272,1(1986).

[5] T.Plehn, M.Spira and P.M. Zerwas, Nucl. Phys. B479,46(1996).

[6] D.Bowser-Chao, K.Cheung, and S.Thomas, Phys. Lett B315, 399(1993); Ma WenGan, Chong-Sheng Li and Hang Liang, Phys. Rev. D53,1304(1996),[E:1997, Phys. Rev. D56, 4420].

[7] Jiang Yi, Han Liang, Ma Wen-Gan, Yu Zeng-Hui and Han Meng, J. of Phys. G23,385(1997), [E:1997, J. Phys. G: Nucl. Phys. 23, 1151].

[8] R.Arnowitt, A.H. Chamseddine and P.Nath, Phys. Rev. Lett, 49,970(1982); 50,232(1983); Phys. Lett. B121,33(1983); H.P.Nilles, Phys. Rep. 110,1(1984); J. Ellis, C.Kounnas and D.V. Nanopoulos, Nucl. Phys. B241,406(1984). 
[9] Scott.S.D.Willenbrock, Phys. Rev. D 35,173(1987).

[10] A. Krause, T Plehn, M. Spira and P.M.Zerwas, 'Pair production of charged Higgs boson pairs in gluon-gluon collisions', hep-ph/9707430.

[11] J. Ellis and S. Rudaz, Phys. Lett. B128,248(1983); F. Gunion and H.E. Haber, Nucl. Phys. bf B272,1(1986).

[12] H.E. Haber, G.L. Kane, Phys. Rep. 117(1985)75.

[13] M. Gell-Mann, Phys. Rev. 125,1067(1962).

[14] A.D. Martin, W.J. Stirling and R.G. Roberts, Phys. Lett.B354, 155(1995).

[15] Particle Data Group, Phys. Rev. D 50, No.3(1994); D. Schaile, CERN-PPE/94-162(11 October 1994).

[16] Bernd A. Kniehl, Phys. Rep. 240(1994)211.

[17] G. Passarino and M. Veltman, Nucl. Phys. B160, 151(1979).

[18] J.F. Gunion, H.E. Haber, G. Kane and S. Dawson, The Higgs Hunter's Guide, Addison Wesley, Reading, MA(1990);

\section{Figure captions}


Fig.1 The Feynman diagrams of subprocess $g g \rightarrow H^{+} H^{-}$. Figures $(1 \sim 10)$ are so called THDM diagrams. Figures $(11 \sim 32)$ are the diagrams having one squark-loop. $(1 \sim 4$, $11 \sim 20,27 \sim 32)$ are s-channel diagrams. The others are box diagrams which include tand u-channels.

Fig.2(1) Total cross sections of the subprocess $g g \rightarrow H^{+} H^{-}$as the functions of the $\sqrt{\hat{s}}$ with $m_{H^{ \pm}}=150 \mathrm{GeV}$ in the MSSM. The full-line is for $\tan \beta=1.5$. The dotted-line is for $\tan \beta=30$

Fig.2(2) Total cross sections of the subprocess $g g \rightarrow H^{+} H^{-}$as the functions of the $\sqrt{\hat{s}}$ with $m_{H^{ \pm}}=300 \mathrm{GeV}$ in the MSSM. The full-line is for $\tan \beta=1.5$. The dotted-line is for $\tan \beta=30$.

Fig.3 Total cross sections of the subprocess $g g \rightarrow H^{+} H^{-}$as the functions of the $\tan \beta$ with $m_{H^{ \pm}}=150 \mathrm{GeV}$ in the MSSM. The full-line and dotted-line correspond to $\sqrt{\hat{s}}=400 \mathrm{GeV}$ and $\sqrt{\hat{s}}=800 \mathrm{GeV}$ respectively.

Fig.4 Total cross sections of the subprocess $g g \rightarrow H^{+} H^{-}$as the functions of the $m_{H^{ \pm}}$with $\sqrt{\hat{s}}=1 \mathrm{TeV}$ in MSSM. The full-line is for $\tan \beta=1.5$. The dotted-line is for $\tan \beta=30$.

Fig.5(1) Total cross sections of the process $p p \rightarrow g g \rightarrow H^{+} H^{-}+X$ as the functions of the $\sqrt{s}$ with $m_{H^{ \pm}}=150 \mathrm{GeV}$ at the LHC energy region. The full-line is for $\tan \beta=1.5$ in the MSSM. The dashed-line is for $\tan \beta=1.5$ in the THDM. The dotted-line is for $\tan \beta=30$ in the MSSM. The dashed-dotted-line is for $\tan \beta=30$ in the THDM. 
Fig.5(2) Total cross sections of the process $p p \rightarrow g g \rightarrow H^{+} H^{-}+X$ as the functions of the $\sqrt{s}$ with $m_{H^{ \pm}}=300 \mathrm{GeV}$ at the LHC energy region. The full-line is for $\tan \beta=1.5$ in the MSSM. The dashed-line is for $\tan \beta=1.5$ in the THDM. The dotted-line is for $\tan \beta=30$ in the MSSM. The dashed-dotted-line is for $\tan \beta=30$ in the THDM. 


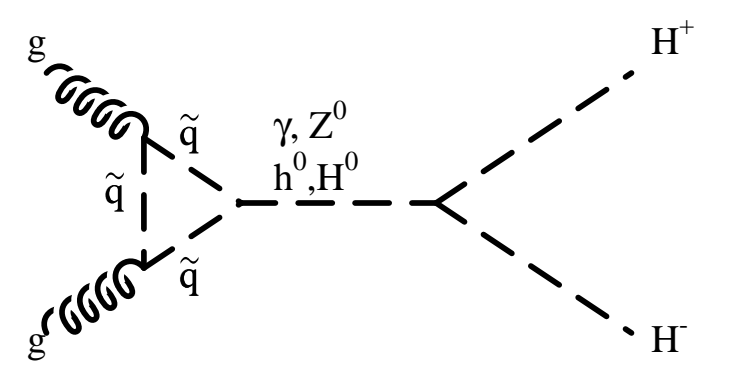

$(11)(12)(13)(14)$

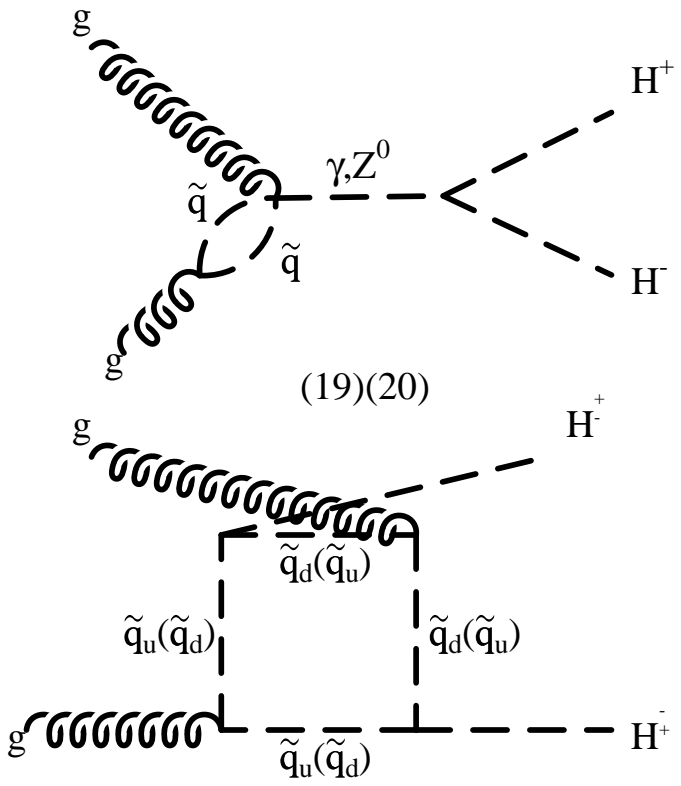

(25)(26)

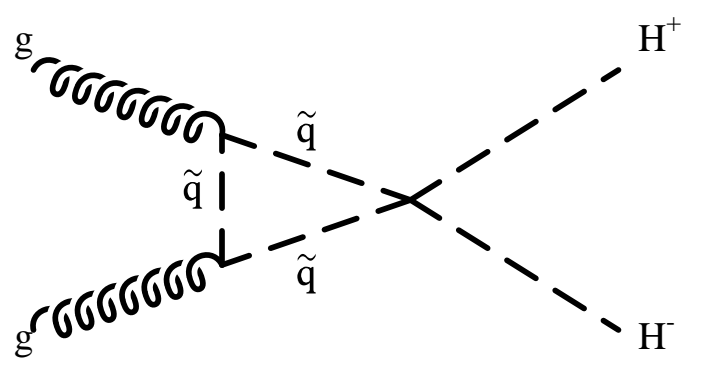

(29)(30)

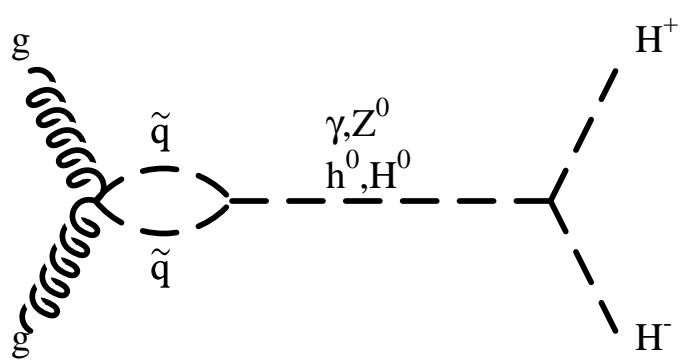

$(15)(16)(17)(18)$

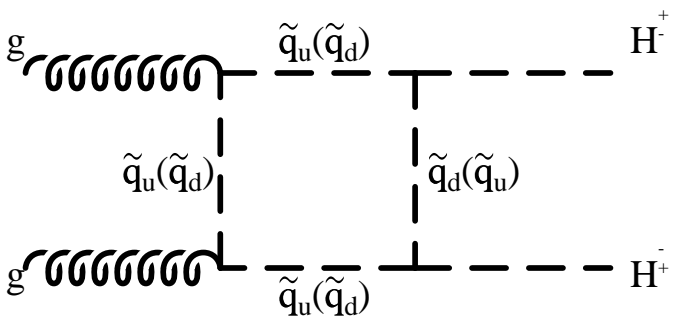

(21)(22)(23)(24)

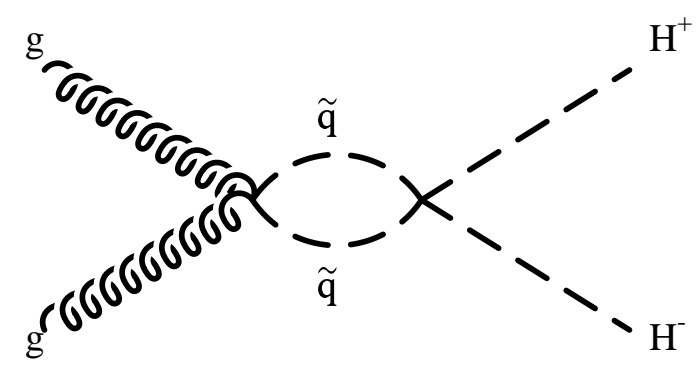

(27)(28)

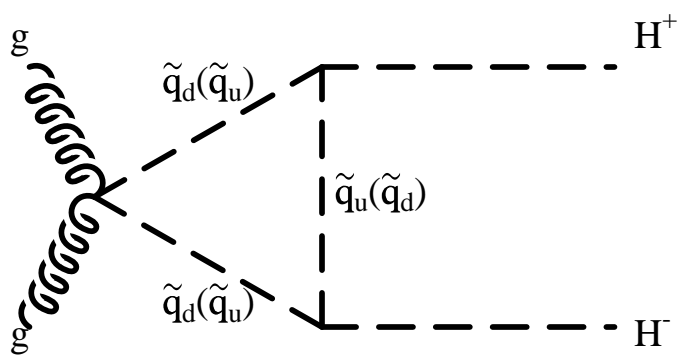

(31)(32)

Fig.1 


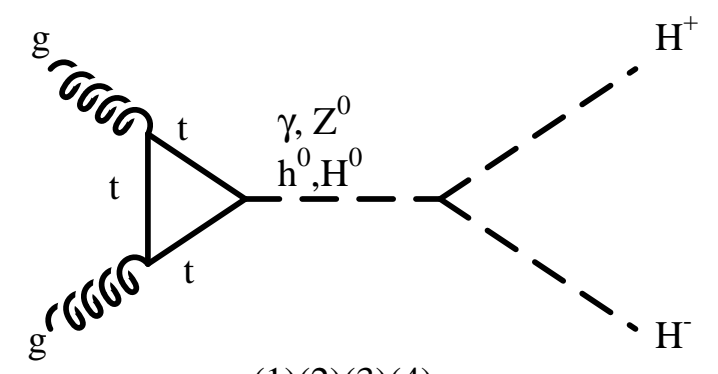

(1)(2)(3)(4)

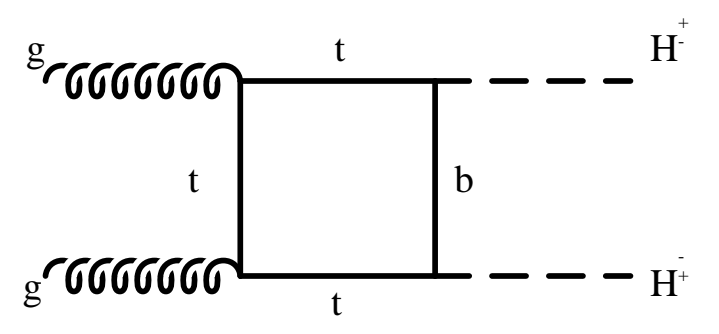

(5)(6)

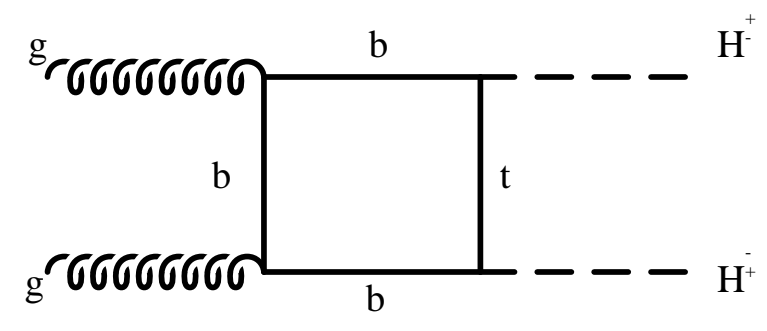

(7)(8)

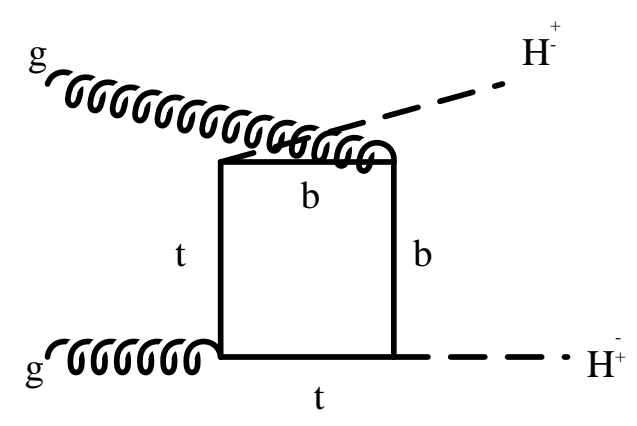

(9)(10)

Fig.1 
Fig.3

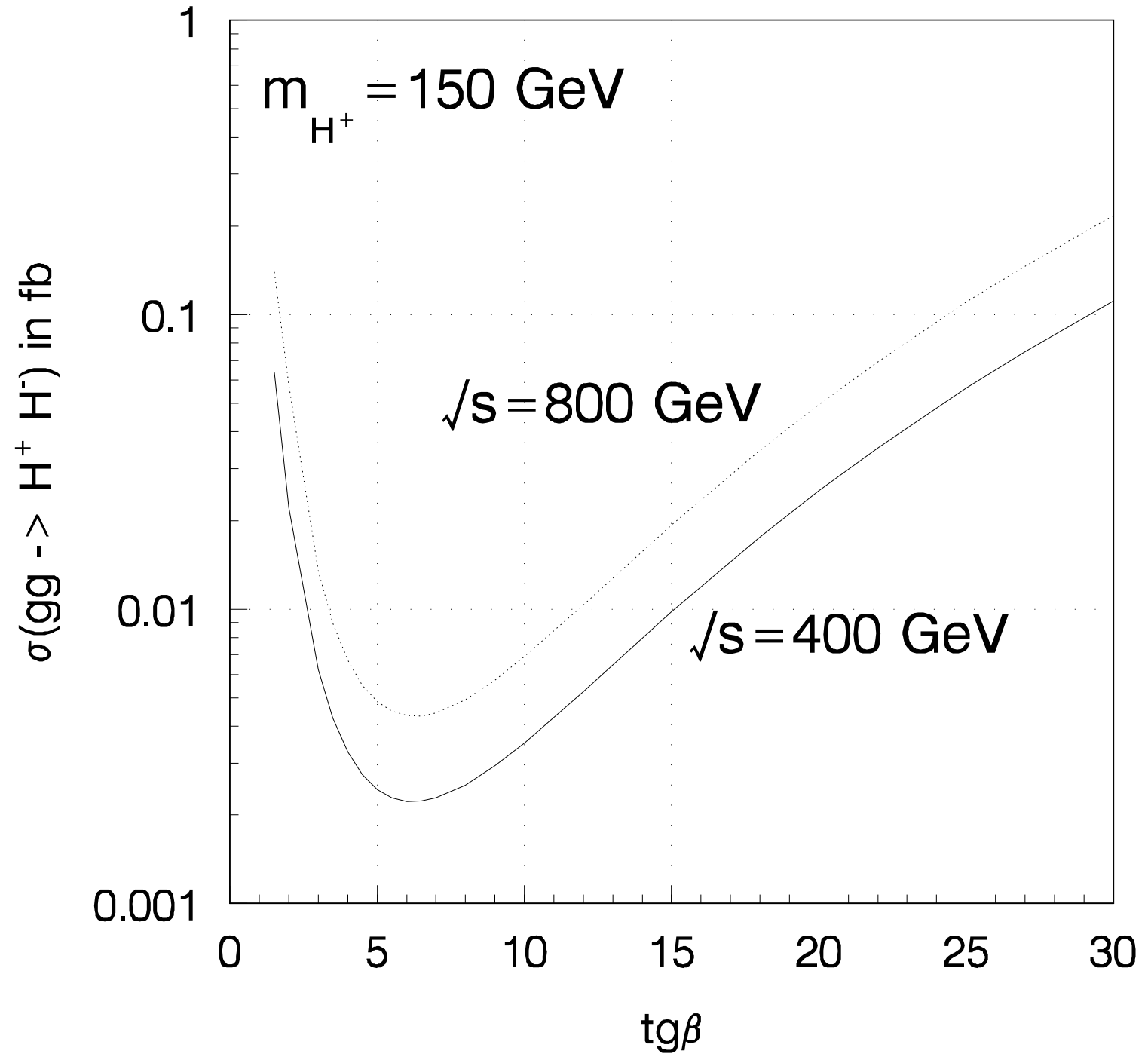


Fig.4

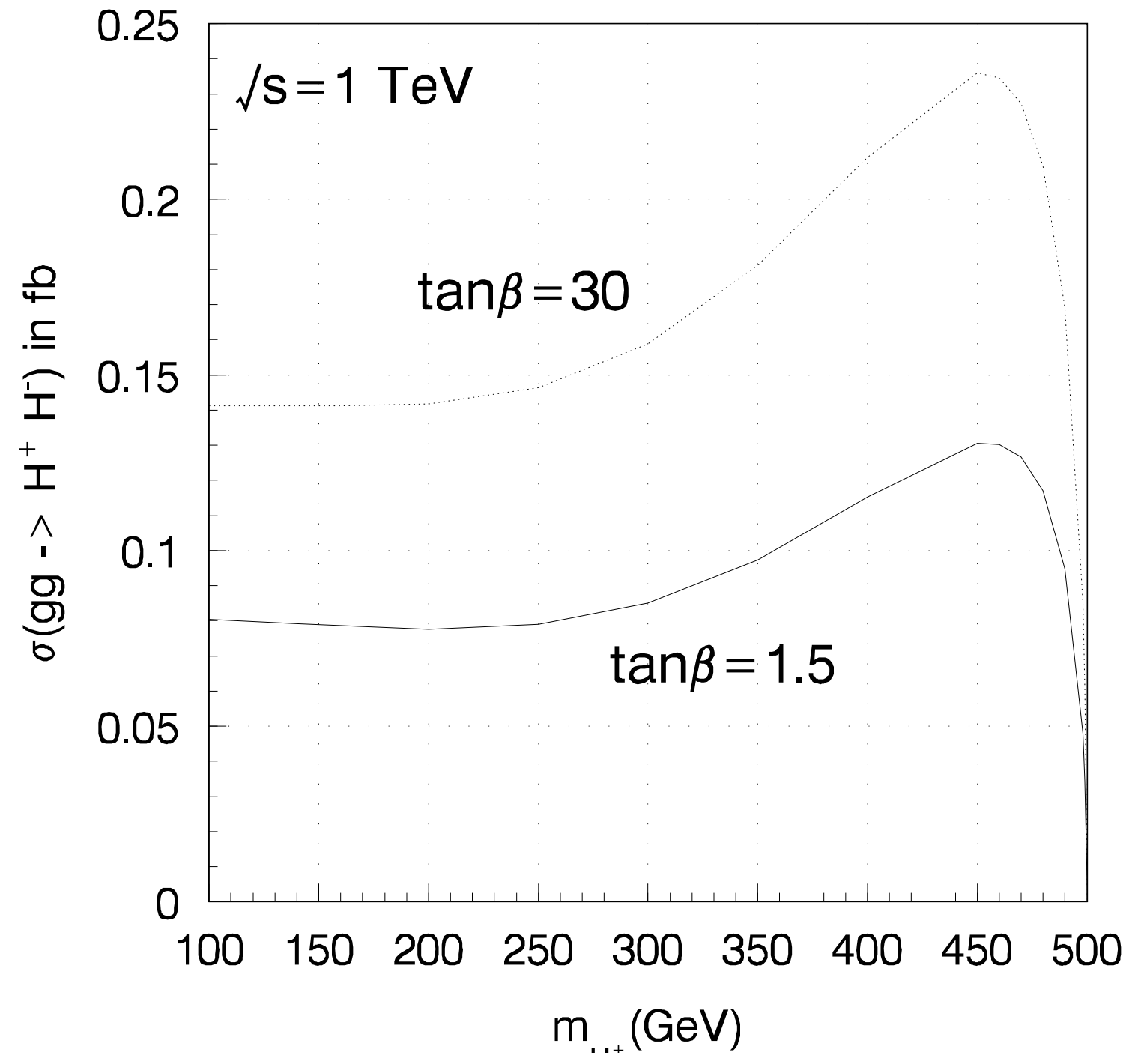


Fig.2(1)

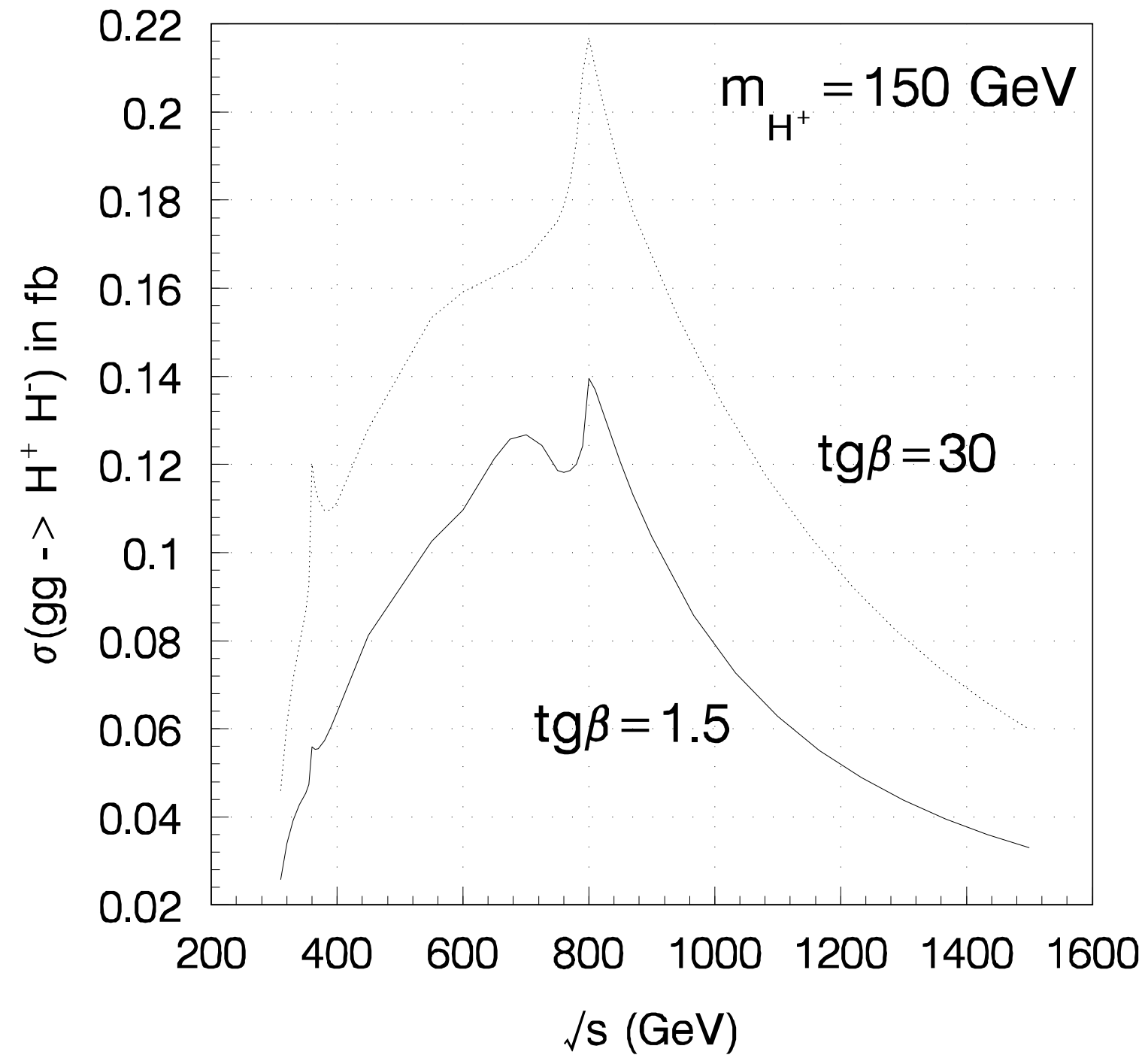


Fig.2(2)

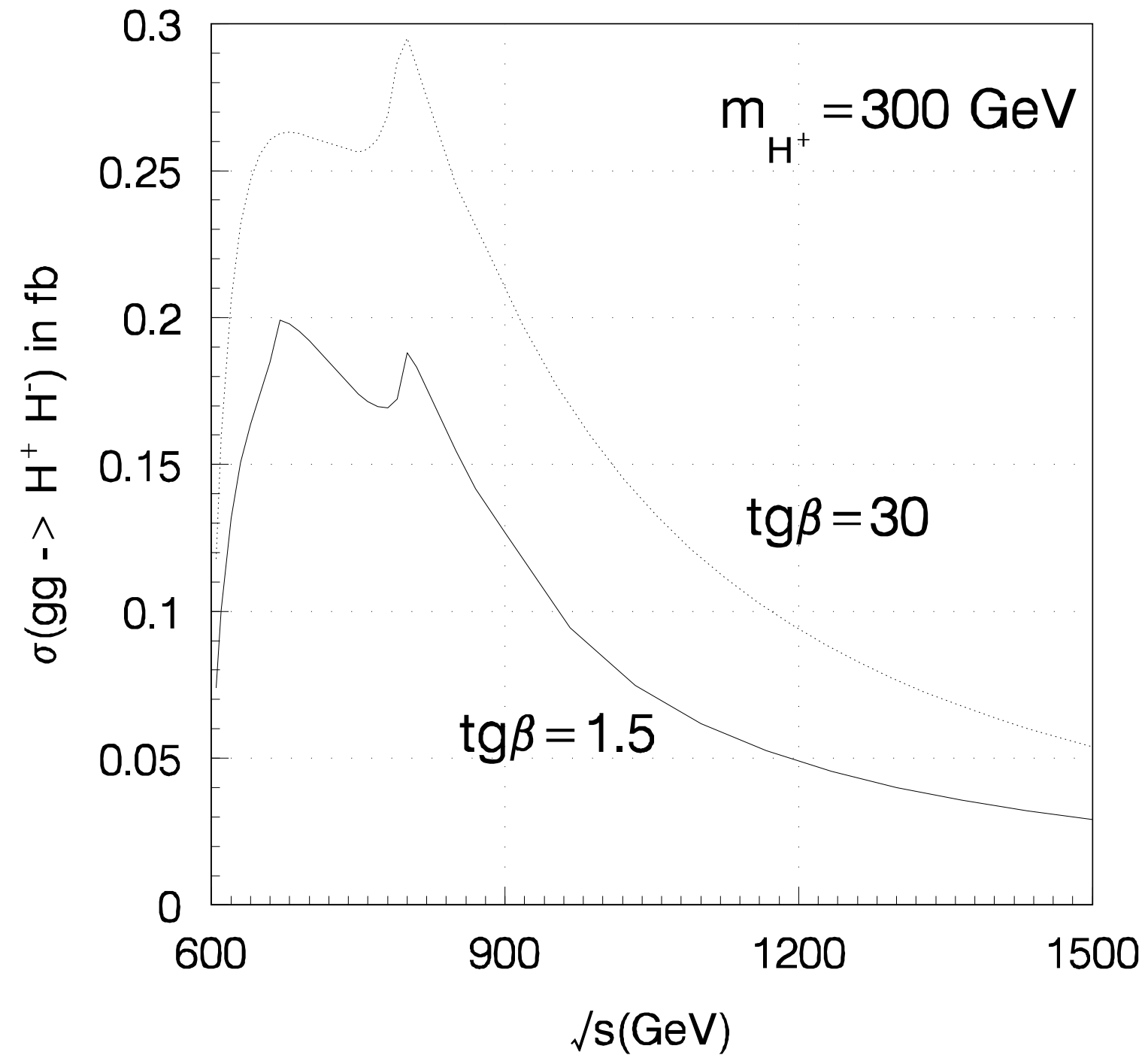


Fig.5(1)

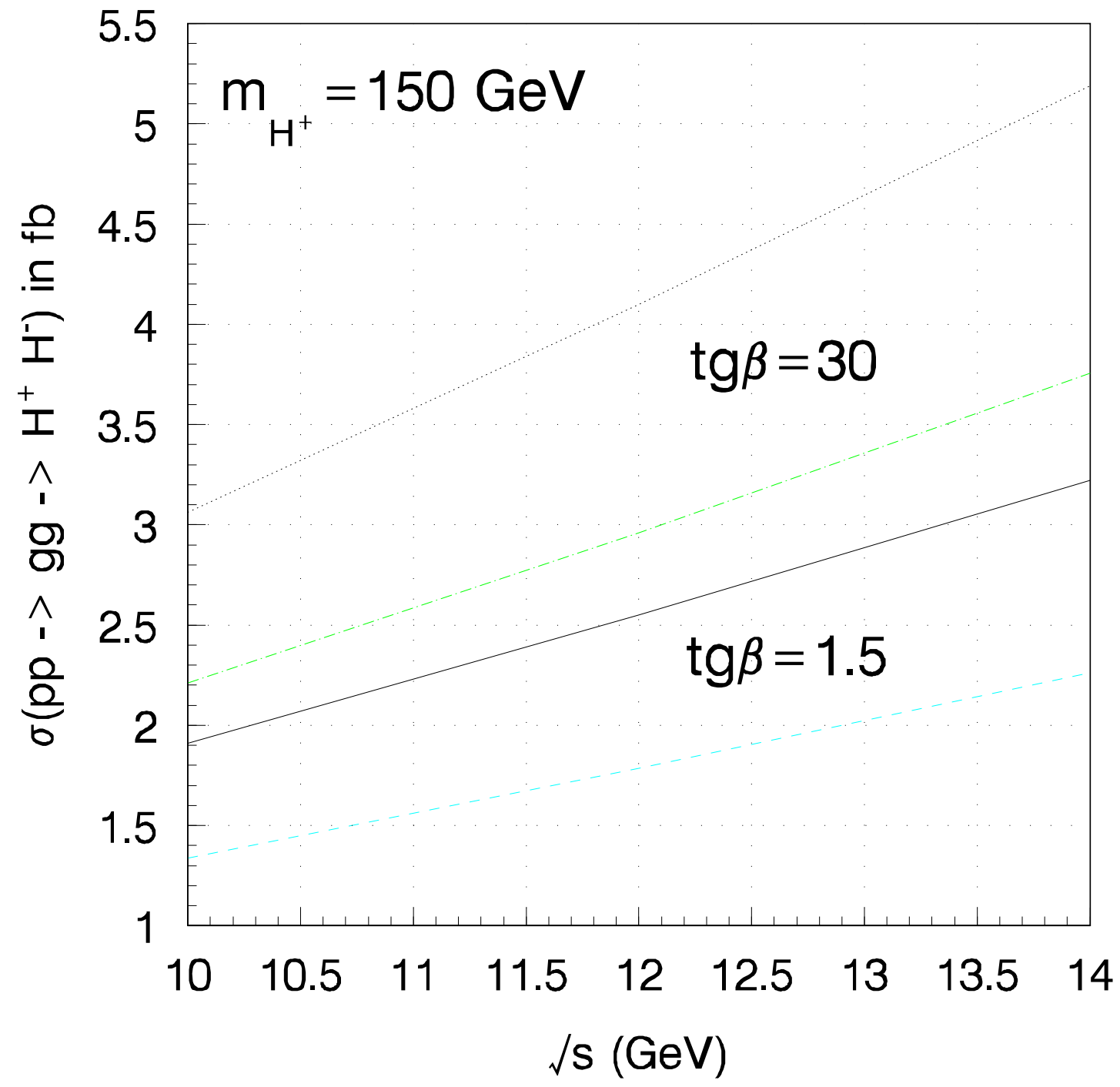


Fig.5(2)

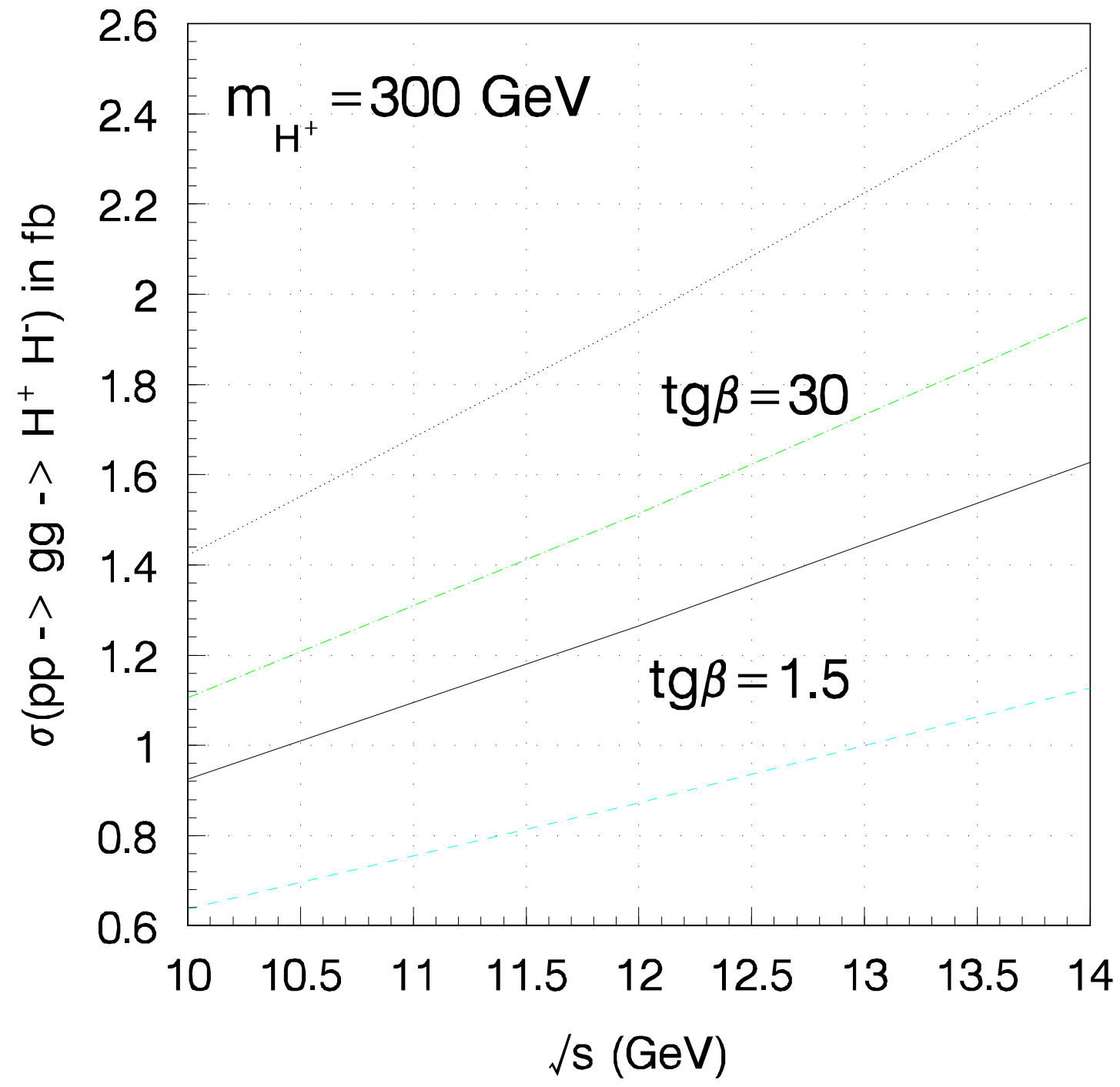

